\title{
Atividade física e fatores associados em doentes arteriais coronarianos
}

\author{
Physical activity and associated factors in patients with coronary artery disease
}

\author{
Marcelo Cozzensa da Silva ${ }^{1}$, Josi Mara Saraiva de Oliveira ${ }^{2}$, Leonardo Alves ${ }^{3}$, Alice Meyer lepsen ${ }^{4}$ \\ ${ }^{1}$ Doutor em Epidemiologia pela Universidade Federal de Pelotas (UFPEL), Professor do Programa de Pós-Graduação em Educação Física da UFPEL, Membro do Grupo \\ de Estudos em Epidemiologia da Atividade Física (GEEAF), Pelotas, RS; ${ }^{2}$ Mestre em Educação Física pela UFPEL, Especialista em Atividade Física, Desempenho Motor e \\ Saúde, pela Universidade Federal de Santa Maria, Santa Maria, RS; ${ }^{3}$ Mestre em Epidemiologia pela UFPEL, Professor da Faculdade de Medicina da Universidade Federal
} do Rio Grande (FURG), Rio Grande, RS; ${ }^{4}$ Mestranda do Programa de Pós-Graduação em Educação Física da UFPEL.

Este trabalho é parte do projeto da dissertação de mestrado "Intenção e Comportamento da Prática Regular de Atividades Físicas e Fatores Associados em Indivíduos com Doença Arterial Coronariana", defendida por JMSO e orientada por MCS, na Escola Superior de Educação Física da UFPEL, disponível na página eletrônica do Programa de Pós-Graduação em Educação Física da UFPEL. Apoio financeiro do Conselho Nacional de Desenvolvimento Científico e Tecnológico (CNPq).

\section{RESUMO}

Objetivos: Identificar a frequência e os fatores associados à prática de atividade física em portadores de doença arterial coronariana, durante os seis meses prévios à hospitalização por evento isquêmico agudo.

Métodos: Foi realizado um estudo transversal em indivíduos adultos diagnosticados com doença arterial coronariana não complicada por angina instável e/ou infarto do miocárdio, internados no Hospital de Cardiologia da Santa Casa de Rio Grande, Rio Grande do Sul, Brasil. O questionário incluiu variáveis sociodemográficas (sexo, idade, cor da pele e situação conjugal) econômicas (renda e escolaridade), comportamental (tabagismo atual), nutricional (índice de massa corporal), morbidades existentes (hipertensão arterial e dislipidemia), percepção de saúde e prática de atividade fisica no lazer nos seis meses que antecederam a hospitalização.

Resultados: Durante o período de cinco meses de recrutamento, 150 pacientes foram entrevistados. No período de seis meses anteriores à internação hospitalar, $25,3 \%$ dos avaliados relataram realizar atividade física três vezes por semana por pelo menos 30 minutos diários. A prática esteve associada na análise bruta com as variáveis renda familiar e hipertensão arterial. Na análise ajustada o desfecho permaneceu associado à renda familiar, ocorrendo uma redução da prática à medida que aumentava a renda dos indivíduos.

Conclusões: Na amostra estudada, a frequência da prática regular de atividade física previamente à ocorrência de um evento coronariano agudo foi baixa e apresentou uma tendência de redução à medida que a renda familiar aumentava.

DESCRITORES: ATIVIDADE MOTORA; ATIVIDADE FÍSICA; DOENÇAS CARDIOVASCULARES; ESTUDOS TRANSVERSAIS.

\section{ABSTRACT}

Aims: To identify the frequency and factors associated with physical activity in patients with coronary artery disease during the six months prior to hospitalization for an acute ischemic event.

Methods: A cross-sectional study was conducted in adults diagnosed with coronary artery disease without unstable angina and/or myocardial infarction, admitted to the Cardiology Hospital of Santa Casa de Rio Grande, Rio Grande do Sul, Brazil. The questionnaire included sociodemographic variables (gender, age, race and marital status), economic (income and education), behavioral (current smoking), nutrition (body mass index), existing morbidities (systolic hypertension and dyslipidemia), health perception and practice of physical activity during leisure time in the six months prior to hospitalization.

Results: During the five months of recruitment, 150 patients were interviewed. In the period of six months prior to hospitalization, $25.7 \%$ of the patients reported physical activity three times a week for at least 30 minutes. The practice was associated in the crude analysis with the variables family income and hypertension. In the adjusted analysis, the outcome remained associated with family income, a reduction of the practice occurring with increasing of the individuals income.

Conclusions: In this sample, the frequency of regular physical activity prior to the occurrence of an acute coronary event was low and tended to decrease as family income increased.

KEY WORDS: MOTOR ACTIVITY; PHYSICAL ACTIVITY; CARDIOVASCULAR DISEASES; CROSS-SECTIONAL STUDIES. 


\section{INTRODUÇÃO}

A doença arterial coronariana (DAC) é importante causa de incapacidade e morte prematura em todo o mundo e representa cerca de $30 \%$ das mortes por todas as causas. ${ }^{1}$ A previsão para o ano de 2020 é de que mais de $40 \%$ do total dos óbitos da população mundial estarão relacionados às doenças cardiovasculares e, em 2030, a previsão é que o número de mortes ultrapassará 24 milhões por ano. ${ }^{2}$

A atividade física regular é recomendada para pessoas com DAC como um meio de prevenir e melhorar inúmeros fatores de risco a ela associados. ${ }^{3,4}$ Estudos têm mostrado consistentemente que o aumento da atividade física após um evento cardíaco está associado ao aumento da capacidade do exercício, ${ }^{5}$ sendo esse um dos mais fortes indicadores prognósticos na prevenção de novos eventos. ${ }^{6}$ Apesar de conhecidos os benefícios da atividade física regular nessa população, poucos pacientes são ativos o suficiente para obter benefícios à saúde. ${ }^{7}$

Identificar a frequência de prática regular de atividade física e os fatores a ela associados entre indivíduos acometidos por doenças crônicas, especialmente entre aqueles acometidos por DAC, tem sido um desafio entre os pesquisadores da área de atividade física. A determinação dessa prevalência e dos fatores a ela associados é de fundamental importância para a prevenção e tratamento dessa morbidade.

Neste contexto, o presente estudo teve o objetivo de identificar a ocorrência e os fatores associados à prática de atividade física em portadores de DAC em período anterior à hospitalização por evento isquêmico agudo.

\section{MÉTODOS}

Este estudo caracteriza-se por ser do tipo transversal, tendo os participantes sido entrevistados e avaliados durante internação hospitalar. O estudo foi submetido e aprovado pelo Comitê de Ética em Pesquisa da Escola Superior de Educação Física da Universidade Federal de Pelotas sob número 121/2010 e as entrevistas foram realizadas após o consentimento por escrito dos entrevistados, aos quais foi garantido o sigilo das informações e esclarecimento de possíveis dúvidas.

Participaram da pesquisa indivíduos adultos com idade entre 30 e 79 anos, de ambos os sexos, portadores de DAC não complicada por angina instável e/ou infarto agudo do miocárdio, internados no Hospital de Cardiologia da Santa Casa de Rio
Grande, Rio Grande do Sul, referência regional no sul do Brasil. Todos os casos elegíveis para o estudo foram selecionados consecutivamente durante a internação hospitalar entre os meses de junho a outubro de 2010. Aqueles considerados inaptos à prática regular de atividade física por problemas motores físicos (que os incapacitassem de realizar atividade física no período anterior ao evento coronariano) e/ou problemas psíquicos (incapacitantes para responder ao questionário) foram excluídos do estudo. Este critério de exclusão foi necessário porque um dos objetivos do grande estudo no qual esta pesquisa estava inserida era o de verificar a intenção de praticar atividade física na alta hospitalar, bem como a prática em períodos subsequentes a ela.

Os indivíduos foram contatados por entrevistadoras previamente treinadas para a coleta de dados. As entrevistas foram realizadas de forma individual durante a internação hospitalar. Realizou-se a aplicação de um questionário testado e pré-codificado, contendo informações demográficas (idade: anos completos; sexo: masculino, feminino; cor da pele: branco, não branco), socioeconômicas (escolaridade: anos de escolaridade; renda familiar: em salários mínimos; situação conjugal: casado, solteiro, separado, viúvo), comportamental (tabagismo: fuma, ex-fumante, nunca fumou), nutricional (índice de massa corporal (IMC): medido pelo peso em quilos dividido pela altura em centímetros elevada ao quadrado, ambos autoreferidos), diagnóstico de morbidades (hipertensão arterial: não, sim; dislipidemia: não, sim), percepção de saúde (excelente, muito bom, bom, regular, ruim) e prática de atividade fisica no lazer (menos de uma vez por semana, uma vez por semana, duas vezes por semana, três ou mais vezes por semana).

Foram considerados ativos aqueles indivíduos que, no período de seis meses antecedentes ao evento cardíaco, relataram a prática regular de qualquer atividade física realizada no domínio do lazer por no mínimo 30 minutos, três vezes na semana.

O banco de dados foi construído no programa Epi Info 3.5.1 for Windows e a análise realizada no programa STATA 10.0. ${ }^{8}$ A análise bivariada examinou tabelas de contingência e a associação estatística foi aferida para valor $\mathrm{p}<0,05$ pelos testes de qui quadrado de Pearson para heterogeneidade ou tendência linear. A análise multivariável foi realizada através de regressão de Poisson, que permitiu controle simultâneo de fatores que levaram em conta a hierarquia de determinação da prática de atividade física.

O modelo proposto para a hierarquia citada foi constituído de quatro níveis para o desfecho prática 
de atividade física: o primeiro, em que estão inseridas as variáveis demográficas e econômicas (sexo, idade e cor da pele, renda, escolaridade e situação conjugal), o segundo que abrange as variáveis comportamental (fumo) e nutricional (IMC), o terceiro abrangendo morbidades existentes (hipertensão arterial e dislipidemia) e, por último, em um quarto estágio, a percepção de saúde dos indivíduos.

Os efeitos das variáveis do primeiro nível foram controlados entre si; as do segundo nível foram controlados entre elas e para as do primeiro nível; as do terceiro nível foram controladas entre elas e para as dos dois níveis anteriores, a do quarto nível entre elas e para os níveis anteriores, e a do quinto nível (desfecho) para os demais níveis precedentes. ${ }^{9}$ Entraram no modelo hierarquizado de análise todas as variáveis independentemente do valor $p$ encontrado na análise bruta. As variáveis que na análise multivariável apresentaram valor $\mathrm{p} \leq 0,2$ permaneceram na análise sempre que preenchiam os critérios para prováveis fatores de confusão. Ficaram no modelo final todas as variáveis que apresentaram valor $\mathrm{p}<0,05$.

\section{RESULTADOS}

Durante o período de cinco meses de recrutamento, 150 indivíduos foram entrevistados. A média de idade

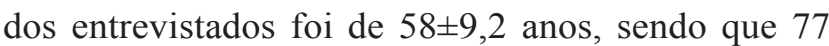
$(51,3 \%)$ eram do sexo masculino e $134(89,3 \%)$ tinham a cor da pele branca. Quanto à escolaridade, $96(70,7 \%)$ tinham até ensino fundamental e 44 (29,3\%) tinham pelo menos ensino médio. Cento e dois participantes (68\%) eram casados ou viviam com companheiro e metade da amostra, 73 pacientes $(51,4 \%)$, viviam com renda familiar de até dois salários mínimos. Os dados demográficos, socioeconômicos, comportamentais e nutricional da amostra podem ser observados na Tabela 1.

$\mathrm{O}$ número de indivíduos que praticavam atividade física regular antes do evento isquêmico que determinou a sua internação foi de 38 (25,3\%; IC95\% 18,8 a 33,5\%). $\mathrm{Na}$ análise bruta observou-se que a prática regular de atividade física apresentou associação inversa com a renda familiar dos indivíduos, bem como foi mais elevada entre os que apresentavam hipertensão arterial. $\mathrm{Na}$ análise ajustada a prática de atividade física permaneceu associada a renda familiar dos indivíduos, sendo menor a medida que a renda aumentava. A associação entre o desfecho e a variável hipertensão arterial perdeu significância após controle para as demais variáveis levadas em consideração na análise ajustada (Tabela 2).
Tabela 1. Descrição da amostra dos 150 pacientes com doença arterial coronariana entrevistados durante a internação hospitalar por evento isquêmico agudo. Hospital de Cardiologia da Santa Casa de Rio Grande. Rio Grande, RS.

\begin{tabular}{|c|c|c|}
\hline Variáveis & $\mathbf{N}$ & $\%$ \\
\hline \multicolumn{3}{|l|}{ Sexo } \\
\hline Feminino & 73 & 48,7 \\
\hline Masculino & 77 & 51,3 \\
\hline \multicolumn{3}{|l|}{ Idade } \\
\hline Até 49 anos & 25 & 16,7 \\
\hline 50-59 anos & 59 & 39,3 \\
\hline $60-69$ anos & 48 & 32,0 \\
\hline 70 ou mais & 18 & 12,0 \\
\hline \multicolumn{3}{|l|}{ Cor da pele } \\
\hline Branca & 134 & 89,3 \\
\hline Não Branca & 16 & 10,7 \\
\hline \multicolumn{3}{|l|}{ Renda Familiar (salário mínimo) ${ }^{\mathrm{a}}$} \\
\hline Até 1 Salário & 42 & 29,6 \\
\hline Entre 1 e 2 Salários & 31 & 21,8 \\
\hline Entre 2 e 3 Salários & 18 & 12,7 \\
\hline Entre 3 e 4 Salários & 21 & 14,8 \\
\hline 4 ou mais Salários & 30 & 21,1 \\
\hline \multicolumn{3}{|l|}{ Escolaridade } \\
\hline Sem escolaridade & 07 & 4,7 \\
\hline Fundamental & 99 & 66,0 \\
\hline Ensino Médio & 37 & 24,7 \\
\hline Ensino Superior & 07 & 4,6 \\
\hline \multicolumn{3}{|l|}{ Situação Conjugal } \\
\hline Solteiro/Separado/Viúvo & 48 & 32,0 \\
\hline Casado/Vive com Companheiro & 102 & 68,0 \\
\hline \multicolumn{3}{|c|}{ Classificação do estado nutricional pelo $\mathrm{IMC}^{\mathrm{b}}$} \\
\hline Normal & 41 & 27,5 \\
\hline Sobrepeso & 63 & 42,3 \\
\hline Obesidade & 45 & 30,2 \\
\hline \multicolumn{3}{|l|}{ Tabagismo } \\
\hline Não Fumante & 71 & 47,3 \\
\hline Fumante & 34 & 22,7 \\
\hline Ex-Fumante & 45 & 30,0 \\
\hline \multicolumn{3}{|l|}{ Hipertensão arterial } \\
\hline Não & 45 & 30,0 \\
\hline Sim & 105 & 70,0 \\
\hline \multicolumn{3}{|l|}{ Dislipidemia } \\
\hline Não & 78 & 52,0 \\
\hline Sim & 72 & 48,0 \\
\hline \multicolumn{3}{|l|}{ Percepção de saúde } \\
\hline Muito bom/bom & 30 & 20,0 \\
\hline Regular & 92 & 61,3 \\
\hline Ruim & 28 & 18,7 \\
\hline
\end{tabular}

a Salário Mínimo Nacional; bíndice de Massa Corporal. 
Tabela 2. Prevalência de prática regular de atividade física prévia em 150 pacientes com doença arterial coronariana entrevistados durante a internação hospitalar por evento isquêmico agudo. Análise bruta e ajustada entre prática de atividade física e variáveis independentes em estudo. Rio Grande, RS.

\begin{tabular}{|c|c|c|c|c|c|}
\hline \multirow{2}{*}{ Variáveis } & \multirow{2}{*}{$\%$} & \multicolumn{2}{|c|}{ Análise bruta } & \multicolumn{2}{|c|}{ Análise ajustada } \\
\hline & & RP (IC 95\%) & Valor $\mathbf{p}$ & RP (IC 95\%) & Valor $p$ \\
\hline $\operatorname{Sexo}^{1}$ & & & $0,9^{*}$ & & \\
\hline Feminino & 26,0 & 1,0 & & & \\
\hline Masculino & 25,3 & $1,0(0,6$ a 1,7$)$ & & & \\
\hline Idade $^{1}$ & & & $0,5^{* *}$ & & \\
\hline Até 49 anos & 12,0 & 1,0 & & & \\
\hline 50-59 anos & 31,0 & $2,6(0,8$ a 8,0$)$ & & & \\
\hline $60-69$ anos & 27,7 & $2,3(0,7$ a 7,4$)$ & & & \\
\hline 70 anos ou mais & 22,2 & $1,8(0,5$ a 7,3$)$ & & & \\
\hline Cor da pele ${ }^{1}$ & & & $0,9 *$ & & \\
\hline Branco & 25,8 & 1,0 & & & \\
\hline Não branco & 25,0 & $1,0(0,4$ a 2,4$)$ & & & \\
\hline Renda familiar (salário mínimo)²a & & & $0,04 * *$ & & $0,04^{* *}$ \\
\hline Até 1 Salário & 42,9 & 1,0 & & 1,0 & \\
\hline Entre 1 e 2 Salários & 23,3 & $0,5(0,3$ a 1,1$)$ & & $0,5(0,3$ a 1,1$)$ & \\
\hline Entre 2 e 3 Salários & 11,1 & $0,3(0,1$ a 1,0$)$ & & $0,3(0,1$ a 1,0$)$ & \\
\hline Entre 3 e 4 Salários & 25,0 & $0,6(0,2$ a 1,3$)$ & & $0,6(0,2$ a 1,3$)$ & \\
\hline 4 ou mais Salários & 18,5 & $0,4(0,2$ a 1,0$)$ & & $0,4(0,2$ a 1,0$)$ & \\
\hline Escolaridade $^{2}$ & & & $0,5^{* *}$ & & \\
\hline Sem escolaridade & 42,9 & 1,0 & & & \\
\hline Primário & 24,7 & $0,6(0,2$ a 1,5$)$ & & & \\
\hline Secundário & 27,0 & $0,6(0,2$ a 1,7$)$ & & & \\
\hline Superior & 14,3 & $0,3(0,1$ a 2,5$)$ & & & \\
\hline Situação conjugal ${ }^{2}$ & & & $0,3^{*}$ & & \\
\hline Casado/vive com companheiro & 31,3 & 1,0 & & & \\
\hline Solteiro/Separado/Viúvo & 23,0 & $0,7(0,4$ a 1,3$)$ & & & \\
\hline $\mathrm{IMC}^{2 \mathrm{~b}}$ & & & $0,2^{* *}$ & & $0,2^{* *}$ \\
\hline Normal & 21,9 & 1,0 & & 1,0 & \\
\hline Sobrepeso & 21,3 & $1,0(0,5$ a 2,0$)$ & & $1,0(0,5$ a 2,1$)$ & \\
\hline Obesidade & 35,6 & $1,6(0,8$ a 3,3$)$ & & $1,6(0,8$ a 3,3$)$ & \\
\hline Tabagismo $^{3}$ & & & $0,7^{*}$ & & \\
\hline Nunca fumou & 25,7 & 1,0 & & & \\
\hline Fumante atual & 20,6 & $0,8(0,4$ a 1,7$)$ & & & \\
\hline Ex-fumante & 29,6 & $1,1(0,6$ a 2,1$)$ & & & \\
\hline Hipertensão arterial ${ }^{4}$ & & & $0,04 *$ & & $0,08^{*}$ \\
\hline Não & 13,6 & 1,0 & & 1,0 & \\
\hline $\operatorname{Sim}$ & 30,8 & $2,3(1,1$ a 5,0$)$ & & $2,1(0,9$ a 4,7$)$ & \\
\hline Dislipidemia ${ }^{4}$ & & & $0,1^{*}$ & & \\
\hline Não & 20,8 & 1,0 & & & \\
\hline $\operatorname{Sim}$ & 31,0 & $1,5(0,9$ a 2,6$)$ & & & \\
\hline Percepção de saúde ${ }^{4}$ & & & $0,9 *$ & & \\
\hline Muito bom/Bom & 27,6 & 1,0 & & & \\
\hline Regular & 24,2 & $0,9(0,4$ a 1,8$)$ & & & \\
\hline Ruim & 28,6 & $1,0(0,2$ a 2,4$)$ & & & \\
\hline
\end{tabular}

1,2,3,4 Níveis hierárquicos de determinação: ajuste para variáveis do mesmo nível ou nível superior, se valor p<0,2.

* Teste de Wald para heterogeneidade; ** Teste de Wald para tendência.

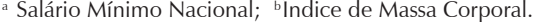

$\mathrm{RP}=$ razão de prevalência; IC 95\%=intervalo de confiança de 95\%. 


\section{DISCUSSÃO}

Neste estudo, a média de idade e de pessoas de cor da pela branca e que vivem com companheiro foi semelhante à encontrada no estudo de Spana et al. ${ }^{10}$ realizado com cardiopatas isquêmicos em uma cidade do interior de São Paulo. O Framingham Heart Study, ${ }^{11}$ reconhecido estudo de coorte sobre fatores de risco e doenças cardiovasculares, mostrava que indivíduos do sexo masculino, de cor da pele branca e mulheres no período pós menopausa, encontram-se em maior risco de eventos cardíacos. ${ }^{11}$ Do total de participantes do presente estudo, 70,7\% tinham até o ensino fundamental, e destes, 4,7\% não tinham escolaridade. Estudo de Favarato et al. ${ }^{12}$ realizado no Instituto do Coração do Hospital das Clínicas da Faculdade de Medicina da Universidade de São Paulo, com portadores de DAC, mostrou que 69,8\% dos pacientes não tinha nem o primeiro grau completo. Fatores de risco para DAC são especialmente mais pronunciados entre pessoas nos níveis socioeconômicos mais baixos. ${ }^{13}$ Embora a maioria dos fatores de risco possam ser prevenidos através de mudanças no estilo de vida que promovam melhora dos hábitos de saúde, ${ }^{14}$ não é claro que pessoas dos níveis econômicos mais baixos atendam a essas mudanças como indivíduos de níveis mais elevados..$^{15} \mathrm{O}$ ambiente que envolve indivíduos de níveis econômicos mais baixos é caracterizado por uma alimentação mais rica em gorduras, elevado stress causado pelo tipo de trabalho que realizam e pelas condições sociais para promoção da saúde. ${ }^{16}$

O fumo é um dos fatores reconhecidamente associados à DAC. ${ }^{17} \mathrm{O}$ CDC (Centers for Disease Control and Prevention), nos Estados Unidos, afirma que fumantes apresentam duas a quatro vezes mais risco de desenvolver doenças cardíacas do que não fumantes, e que o hábito de fumar dobra o risco da pessoa sofrer um infarto. O cigarro duplica o risco para DAC, e 30\% dos casos dessa doença são atribuídos ao número de cigarros fumados. ${ }^{18}$ Os dados disponíveis no Brasil mostram que $40 \%$ dos homens adultos e $25 \%$ das mulheres fumam, sendo que a situação é mais grave nos estados da região Sul. ${ }^{19}$ Apesar dos conhecidos riscos que o hábito de fumar pode trazer à saúde, $23 \%$ da amostra estudada no Hospital de Cardiologia fumava atualmente.

$\mathrm{O}$ excesso de peso e a obesidade são importantes fatores de risco para a DAC. De acordo com a Organização Mundial da Saúde, o número de indivíduos com sobrepeso no mundo aumentará para 1,5 bilhão em 2015 se a tendência atual encontrada, que é de um bilhão, continuar. ${ }^{20} \mathrm{O}$ presente estudo encontrou alta prevalência de indivíduos com IMC referente a sobrepeso/obesidade, correspondente a $72 \%$ da amostra. Em estudo feito no Rio Grande do Sul sobre fatores de risco para a DAC, foi encontrada uma prevalência de $56,3 \%$ de sobrepeso e obesidade, sendo que à medida em que aumentava a faixa etária, aumentavam esses índices. ${ }^{18}$ Outro estudo realizado no mesmo estado, em um hospital universitário da cidade de Porto Alegre, identificou que a obesidade foi o quarto fator de risco mais prevalente entre indivíduos com DAC, estando presente em 28,8\% da amostra. ${ }^{21}$ Estudo de Harris et al. ${ }^{22}$ relatou risco $50 \%$ mais elevado para doenças cardiovasculares entre mulheres com IMC acima de 29, quando comparadas com a população de referência (IMC $<21$ ). Além disso, a obesidade esteve associada com hipertrofia miocárdica, independentemente de hipertensão arterial, e com taxas mais altas de insuficiência cardíaca. ${ }^{23}$ É importante destacar que a obesidade é um problema que, se não controlado, pode ajudar na ocorrência de um novo evento cardíaco. ${ }^{23}$

Dados nacionais estimam que cerca de 20 milhões de pessoas com idade acima de 19 anos apresentem hipertensão arterial sistêmica. ${ }^{24}$ Os dados do estudo atual demonstraram que quase 3/4 dos estudados apresentavam hipertensão arterial, valor muito semelhante ao encontrado em outro estudo realizado no mesmo estado, ${ }^{22}$ no qual a prevalência chegou a $73 \%$. Esse quadro vem sendo demonstrado em vários estudos epidemiológicos e a redução do risco de DCV através do controle da hipertensão arterial foi documentada em inúmeras pesquisas clínicas em prevenção primária. ${ }^{25-27}$ De acordo com o Seven Countries Study ${ }^{28}$ e o Multiple Risk Factor Intervention Trial (MRFIT) ${ }^{29}$ existe uma relação direta entre elevação dos níveis das pressões arteriais sistólica e diastólica e incidência subsequente de mortalidade por DAC. A alta prevalência de hipertensão arterial sistêmica entre esses indivíduos aponta para uma necessidade imediata de atenuação e, principalmente, de prevenção dessa morbidade, pois uma vez que a doença já esteja instalada, pode levar a sérias complicações. ${ }^{30}$

A necessidade de atividade física tem sido apresentada como uma opção importante para a prevenção e tratamento de inúmeras doenças crônicas de alta prevalência em adultos. ${ }^{31}$ Apesar da reconhecida importância da mesma na prevenção primária da doença cardiovascular, a maioria da população adulta brasileira não realiza qualquer atividade física no lazer. Muitas vezes, a divisão entre trabalho, filhos e outras tarefas, pode acabar facilitando a ausência do hábito da prática de atividade física por falta de tempo ou cansaço, entre outros motivos. ${ }^{32}$ Estudos 
conduzidos neste contexto mostram uma prevalência de inatividade física de 71,5\% em Salvador (Bahia), ${ }^{32}$ 66,5\% em Goiânia (Goiás) ${ }^{33}$ e 54,6\% em Florianópolis (Santa Catarina). ${ }^{34}$ Em Pelotas (Rio Grande do Sul), município vizinho ao do presente estudo, a prevalência de inatividade física no lazer foi de $69,8 \%,{ }^{35}$ valor inferior aos $74,3 \%$ dos pacientes que se apresentaram inativos, no domínio do lazer, no período anterior à internação. A prática regular de atividade física no lazer é somente uma das dimensões da atividade física, sendo recomendada a sua realização em vários setores da vida diária, incluindo também atividades ocupacionais, deslocamento e atividades domésticas. ${ }^{36}$ Entretanto, a medida da atividade física no lazer vem ganhando importância, pois apresenta associações mais consistentes com os fatores de risco cardiovasculares. ${ }^{37}$

Entre os estudados, o único fator a permanecer associado à atividade física no lazer foi a renda familiar, apresentando uma relação inversa. Entretanto, grande parte das evidências na literatura científica tem demonstrado uma relação direta entre renda e atividade física, ${ }^{38-40}$ indicando que o estado de pobreza pode ser um complicador no combate à inatividade física. Geralmente indivíduos com nível educacional mais elevado, assim como com maior renda, possuem mais oportunidades, facilidades ${ }^{41}$ e acessos para praticar atividade física, além de conviverem num meio social em que a prática é reconhecida por seus pares como favorável à saúde, o que pode facilitar a adesão. Esses grupos tendem a apresentar melhores condições de saúde, melhor suporte social e assimilam com mais facilidade as recomendações e os benefícios da prática regular de atividade física. ${ }^{38}$

Essas constatações discordam dos resultados deste estudo, em que a presença de atividade física anterior ao evento cardíaco foi mais frequente nas pessoas de menor renda familiar. Não verificamos, entre os estudados, além do quadro cardiológico, quaisquer características que os pudessem diferenciar dos participantes dos outros estudos aqui destacados. Uma hipótese para tal achado poderia estar relacionada à pergunta do questionário que mede a atividade física prévia ao evento cardíaco. Apesar de já utilizada em outros estudos com esse tipo de paciente, a mesma apresenta pouca informação sobre o que pode ser considerada atividade física no lazer, levando a uma superestimativa de indivíduos ativos entre o grupo de menor renda, que tendem a ter profissões menos sedentárias do que os indivíduos de melhor posição socioeconômica.

Este possível viés constitui uma limitação deste estudo. $\mathrm{O}$ auto relato do comportamento da atividade física resulta em dados de pior qualidade quando comparado a um indicador objetivo da atividade física, principalmente pelo fato que muitas pessoas tendem a superestimar seu nível de atividade física. Entretanto, aspectos positivos devem ser destacados na execução desta pesquisa. A medida da atividade física coletada é fundamental e quase inexistente na literatura. Além disso, houve o cuidado metodológico na execução da pesquisa e no tratamento dos dados.

Concluindo, a frequência da prática prévia de atividade física entre indivíduos com DAC avaliados durante a internação hospitalar foi baixa, sendo que pacientes com maior renda familiar apresentam prática de atividade física ainda menor quando comparados aos de menor renda. Sabendo da importância da prática de atividade física como medida de prevenção e tratamento de doenças do sistema cardiocirculatório, é fundamental que políticas públicas sejam implementadas no sentido de estimular a sua prática, o que poderá contribuir para a prevenção das doenças coronarianas. Entretanto a prática de atividade física, principalmente em doentes coronarianos, deve ser precedida por uma avaliação médica, além de ser orientada por educadores físicos capacitados.

\section{AGRADECIMENTOS}

Os autores agradecem ao Conselho Nacional de Desenvolvimento Científico e Tecnológico (CNPq) pelo apoio financeiro, e ao Hospital de Cardiologia da Santa Casa de Rio Grande pela oportunidade de coleta dos dados.

\section{REFERÊNCIAS}

1. WHO. Prevention of cardiovascular disease : guidelines for assessment and management of cardiovascular risk. Geneva: World Health Organization; 2007.

2. Mackay J, Mensah G, Mendis S, Greenlund K, World Health Organization, Dept. of Management of Noncommunicable Diseases. The atlas of heart disease and stroke. Geneva: World Health Organization; 2004.

3. Pina IL, Apstein CS, Balady GJ, Belardinelli R, Chaitman BR, Duscha BD, Fletcher BJ, Fleg JL, Myers JN, Sullivan MJ. Exercise and heart failure: A statement from the American Heart Association Committee on exercise, rehabilitation, and prevention. Circulation. 2003;107(8):1210-25. 
4. Yu CM, Lau CP, Chau J, McGhee S, Kong SL, Cheung BM, Li LS. A short course of cardiac rehabilitation program is highly cost effective in improving long-term quality of life in patients with recent myocardial infarction or percutaneous coronary intervention. Arch Phys Med Rehabil. 2004;85(12):1915-22.

5. Mueller L, Myers J, Kottman W, Oswald U, Boesch C, Arbrol N, Dubach P. Exercise capacity, physical activity patterns and outcomes six years after cardiac rehabilitation in patients with heart failure. Clin Rehabil. 2007;21(10):923-31.

6. Myers J, Prakash M, Froelicher V, Do D, Partington S, Atwood JE. Exercise capacity and mortality among men referred for exercise testing. N Engl J Med. 2002;346(11):793-801.

7. Reid RD, Morrin LI, Pipe AL, Dafoe WA, Higginson LA, Wielgosz AT, LeHaye SA, McDonald PW, Plotnikoff RC, Courneya KS, Oldridge NB, Beaton LJ, Papadakis S, Slovinec D'Angelo ME, Tulloch HE, Blanchard CM. Determinants of physical activity after hospitalization for coronary artery disease: the Tracking Exercise After Cardiac Hospitalization (TEACH) Study. Eur J Cardiovasc Prev Rehabil. 2006;13(4):529-37.

8. StataCorp. Stata statistical software: release 10.0. College Station, TX: StataCorp LP; 2007.

9. Victora CG, Huttly SR, Fuchs SC, Olinto MTA. The role of conceptual frameworks in epidemiological analysis: a hierarchical approach. Int J Epidemiol. 1997;26(1):224-7.

10. Spana TM, Rodrigues RC, Gallani MC, Mendez RD. Physical activity behavior among coronary outpatients according to socio-demographic and clinical profile. Rev Bras Enferm. 2010;63(5):741-8.

11. Dawber TR, Meadors GF, Moore FE: Epidemiological approaches to heart disease: the Framingham Study. Am J Public Health. 1951;41:279.

12. Favarato MECS, Favarato D, Hueb WA, Aldrighi JM. Qualidade de vida em portadores de doença arterial coronária: comparação entre gêneros. Rev Assoc Med Bras. 2006;52(4):236-41.

13. Banks J, Marmot M, Oldfield Z, Smith JP. Disease and disadvantage in the United States and in England. Am Med Assoc. 2006;295:2037-45.

14. Lichtenstein AH, Appel LJ, Brands M, et al. Diet and lifestyle recommendations revision 2006: a scientific statement from the American Heart Association Nutrition Committee. Circulation. 2006;114:82-96.

15. Govil SR, Weidner G, Merritt-Worden T, Ornish D. Socioeconomic status and improvements in lifestyle, coronary risk factors, and quality of life: the Multisite Cardiac Lifestyle Intervention Program. Am J Public Health. 2009;99(7):1263-70.

16. Winkleby MA, Cubbin C, Ahn DK, Kraemer HC.Pathways by which SES and ethnicity influence cardiovascular disease risk factors. Ann N Y Acad Sci. 1999;896:191-209.

17. Fuster V, Gotto AM, Libby P, Loscalzo J, McGill HC. 27th Bethsda Conference - Matching the intensity of risk factor management with the hazard for coronary disease events. Task Force 1. Pathogenesis of coronary disease: the biologic role of risk factors. J Am Coll Cardiol. 1996;27:957-1047.

18. Gus I, Fischmann A, Medina C, Gus M. Prevalência dos Fatores de Risco da Doença Arterial Coronariana no Estado do Rio Grande do Sul. Arq Bras Cardiol. 2002;78(5):478-83

19. Maia CO, Goldmeier S, Moraes MA, Boaz MR, Azzolin K. Fatores de risco modificáveis para doença arterial coronariana nos trabalhadores de enfermagem. Acta Paul Enferm. 2007;20(2):138-42.

20. World Health Organization. The World Health Organization warns of the rising threat of heart disease and stroke as overweight and obesity rapidly increase. [Internet.] [Citado em 4 de abril de 2013.] Disponível em: http://www.who.int/mediacentre/news/release/2005.

21. Feijó MKEF, Lutkmeier R, Ávila CW, Rabelo ER. Fatores de risco para doença arterial coronariana em pacientes admitidos em unidade de hemodinâmica. Rev Gaúcha Enferm. 2009;30(4):641-7.

22. Harris TB, Ballard-Barbasch R, Madans J, Makuc DM, Feldman JJ. Overweight, weight loss, and risk of coronary heart disease in older women: the NHANES I epidemiologic follow-up study. Am J Epidemiol. 1993;137:1318-27.

23. Manson JE, Willett WC, Stampfer MJ. Body weight and mortality among women. N Engl J Med. 1995;333:677-85.

24. Barreto SM, Lotufo P, Pinheiro ARO, Assis AM, Sichieri R, Guimarães V, et al. Análise da Estratégia Global para Alimentação, Atividade Física e Saúde, da Organização Mundial da Saúde. Epidemiol Serv Saúde. 2005;14(1):41-68.

25. Greenlund KJ, Croft JB, Mensah GA. Prevalence of heart disease and stroke risk factors in persons with prehypertension in the United States, 1999-2000. Arch Intern Med 2004;164:2113-2118.

26. National Center for Health Statistics. Health, United States, 2005. With Chartbook on Trends in the Health of Americans. Hyattsville, Md National Center for Health Statistics; 2005. [Citado em outubro de 2012.] Disponível em: http://www.cdc.gov/nchs/data/hus/hus05.pdf.

27. Avezum A, Piegas LS, Pereira JCR. Fatores de risco associados com infarto agudo do miocárdio na região metropolitana de São Paulo. Uma região desenvolvida em um país em desenvolvimento. Arq Bras Cardiol. 2005;84(3):206-13.

28. Keys A. The Seven Countries Study: a multivariate analysis of death and coronary heart disease. Cambridge, MA: Harvard University Press; 1980.

29. Stamler J, Stamler R, Neaton JD. Blood Pressure, systolic and diastolic, and cardiovascular risks: U.S. population data. Arch Intern Med. $1993 ; 153: 598-615$.

30. Veiga EV, Robazzi MLCC, Nogueira MS; Takakura MS, Hayashida M. Estudo dos fatores de risco da hipertensão arterial: conhecimento e exposição. Rev Soc Cardiol Est São Paulo. 1993;6(supl A):1-5.

31. Siqueira FV, Facchini 1LA., Piccini RX, Tomasi E, Thumé E, Silveira DS, Hallal PC. Atividade física em adultos e idosos residentes em áreas de abrangência de unidades básicas de saúde de municípios das regiões Sul e Nordeste do Brasil. Cad Saúde Pública. 2008;24(1):39-54.

32. Pitanga FJG, Lessa I. Prevalência e fatores associados ao sedentarismo no lazer em adultos. Cad Saúde Pública. $2005 ; 21(3): 870-7$. 
33. Peixoto MdRG, Monego ET, Alexandre VP, Souza RGMd, Moura ECd. Monitoramento por entrevistas telefônicas de fatores de risco para doenças crônicas: experiência de Goiânia, Goiás, Brasil. Cad Saude Pública. 2008;24:1323-33.

34. Martins TG, Assis MAAd, Nahas MV, Gauche H, Moura EC. Inatividade física no lazer de adultos e fatores associados. Rev Saúde Pública. 2009;43:814-24

35. Hallal PC, Reichert FF, Siqueira FV, Dumith SC, Bastos JP, da Silva MC, et al. Correlates of leisure-time physical activity differ by bodymass-index status in Brazilian adults. J Phys Act Health. 2008;5(4):571-8

36. Haskell WL, Lee IM, Pate RR, Powell KE, Blair SN, Franklin BA, Macera CA, Heath GW, Thompson PD, Bauman A. Physical activity and public health: updated recommendation for adults from the American College of Sports Medicine and the American Heart Association. Circulation. 2007;116(9):1081-93.

37. Sofi F, Capalbo A, Marcucci R, Gori AM, Fedi S, Macchi C, Casini A, Surrenti C, Abbate R, Gensini GF. Leisure time but not occupational physical activity significantly affects cardiovascular risk factors in an adult population. Eur J Clin Invest. 2007;37(12):947-53.

38. Pan SY, Cameron C, DesMeules M, Morrison H, Craig CL, Jiang XH. Individual, social, environmental, and physical environmental correlates with physical activity among Canadians: a cross-sectional study. BMC Public Health. 2009;9:21.

39. Cerin E, Leslie E. How socio-economic status contributes to participation in leisure-time physical activity. Soc Sci Med. 2008;66:2596-609.

40. de Sousa CA, César CL, Barros MB, Carandina L, Goldbaum M, Marchioni DM, Fisberg RM. Prevalence of leisure-time physical activity and associated factors: a population-based study in São Paulo, Brazil, 2008-2009. Cad Saude Publica. 2013;29(2):270-82.

41. Godin G, Amireault S, Bélanger-Gravel A, Vohl MC, Pérusse L. Prediction of Leisure-time Physical Activity Among Obese Individuals. Obesity. 2009;17(4):706-12. 